\title{
Bank Direct Marketing Analysis of Data Mining Techniques
}

\author{
Hany A. Elsalamony \\ Mathematics Department, Faculty of Science, Helwan University, Cairo, Egypt \\ Computer Science \& Information Department, Arts \& Science College, Salman University, Saudi Arabia
}

\begin{abstract}
All bank marketing campaigns are dependent on customers' huge electronic data. The size of these data sources is impossible for a human analyst to come up with interesting information that will help in the decision-making process. Data mining models are completely helping in the performance of these campaigns. This paper introduces analysis and applications of the most important techniques in data mining; multilayer perception neural network (MLPNN), tree augmented Naïve Bayes (TAN) known as Bayesian networks, Nominal regression or logistic regression (LR), and Ross Quinlan new decision tree model (C5.0). The objective is to examine the performance of MLPNN, TAN, LR and C5.0 techniques on a real-world data of bank deposit subscription. The purpose is increasing the campaign effectiveness by identifying the main characteristics that affect a success (the deposit subscribed by the client) based on MLPNN, TAN, LR and C5.0. The experimental results demonstrate, with higher accuracies, the success of these models in predicting the best campaign contact with the clients for subscribing deposit. The performances are calculated by three statistical measures; classification accuracy, sensitivity, and specificity.
\end{abstract}

\section{General Terms}

Bank Marketing; Data Mining Techniques.

\section{Keywords}

Bank Marketing; Naïve Bayes; Nominal Regression; Neural Network; C5.0.

\section{INTRODUCTION}

In banks, huge data records information about their customers. This data can be used to create and keep clear relationship and connection with the customers in order to target them individually for definite products or banking offers. Usually, the selected customers are contacted directly through: personal contact, telephone cellular, mail, and email or any other contacts to advertise the new product/service or give an offer, this kind of marketing is called direct marketing. In fact, direct marketing is in the main a strategy of many of the banks and insurance companies for interacting with their customers [19].

Historically, the name and identification of the term direct marketing suggested first time in 1967 by Lester Wunderman, which he is considered to be the father of direct marketing [11].

In addition, some of the banks and financial-services companies may depend only on strategy of mass marketing for promoting a new service or product to their customers. In this strategy, a single communication message is broadcasted to all customers through media such as television, radio or advertising firm, etc. [18]. In this approach, companies do not set up a direct relationship to their customers for new-product offers. In fact, many of the customers are not interesting or respond to this kind of sales promotion [20].

Accordingly, banks, financial-services companies and other companies are shifting away from mass marketing strategy because its ineffectiveness, and they are now targeting most of their customers by direct marketing for specific product and service offers $[19,20]$. Due to the positive results clearly measured; many marketers attractive to the direct marketing. For example, if a marketer sends out 1,000 offers by mail and 100 respond to the promotion, the marketer can say with confidence that the campaign led immediately to $10 \%$ direct responses. This metric is known as the 'Response Rate', and it is one of many clear quantifiable success metrics employed by direct marketers. In dissimilarity, general advertising uses indirect measurements, such as awareness or engagement, since there is no direct response from a consumer [11]. From the literature, the direct marketing is becoming a very important application in data mining these days. The data mining has been used widely in direct marketing to identify prospective customers for new products, by using purchasing data, a predictive model to measure that a customer is going to respond to the promotion or an offer [7].

Data mining has gained popularity for illustrative and predictive applications in banking processes. Four techniques will apply to the data set on the bank direct marketing. The Multilayer perception neural network (MLPNN) is one of these techniques, which have their roots in the artificial intelligence. MLPNN is a mutually dependent group of artificial neurons that applying a mathematical or computational model for information processing using a connected approach to computation (Freeman et al., 1991) $[13,24,27]$.

Another technique of data mining is the decision tree approach. Decision tree provides powerful techniques for classification and prediction. There are many algorithms to build a decision tree model $[9,16]$. It can generate understandable rules, and to handle both continuous and categorical variables [23]. One of the famous recent techniques of the decision tree is C5.0, which will be applied in this paper.

A naïve Bayes classifier (TAN) is an easy and simple probabilistic classifier based on applying Bayes' theorem with strong (naïve) independence assumptions. It can predict class membership probabilities, such as the probability that a given sample belongs to a particular class. The assumption is called class conditional independence. It is made to simplify the computation involved and, in this sense, is considered "naïve" [16].

The fourth technique will be using is Logistic regression analysis (LR). Cornfield was the first to use logistic regression in the early $1960 \mathrm{~s}$ and with the wide availability of sophisticated statistical software for high-speed computers; the use of logistic regression is increasing. LR studies the 
association between a categorical dependent and a set of independent (descriptive) fields. The name logistic regression is often used when the dependent variable has only two values. The name multiple-group logistic regression (MGLR) is usually reserved for the case when the dependent variable has three or more unique values. Multiple-group logistic regression is sometimes called multinomial, polytomous, polychotomous, or nominal logistic regression [4].
This paper investigates the effectiveness of four techniques in data mining on the bank direct marketing. They are: back propagation of neural network (MLPNN), naïve Bayes classifier (TAN), Logistic regression analysis (LR), and the recent famous efficient decision tree model (C5.0). The data set is well known as bank marketing from the University of California at Irvine (UCI), as shown in Table 1 [10].

Table 1. ATTRIBUTES

\begin{tabular}{|c|c|c|c|c|c|c|c|c|c|c|c|c|c|c|c|c|c|}
\hline 急 & $\stackrel{8}{4}$ & $\stackrel{\circ}{\circ}$ & 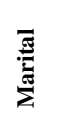 & 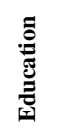 & 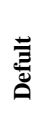 & 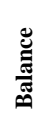 & 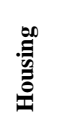 & 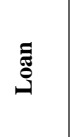 & 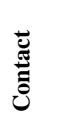 & 胥 & $\frac{\bar{\Xi}}{\frac{\pi}{2}}$ & 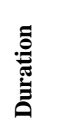 & 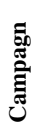 & 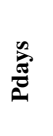 & 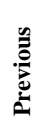 & 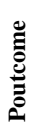 & $\begin{array}{l}\text { 言 } \\
\text { 言 }\end{array}$ \\
\hline$\overline{\underline{\underline{z}}}$ & $\begin{array}{l}\text { 产 } \\
\frac{\bar{z}}{\bar{z}}\end{array}$ & 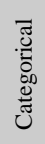 & 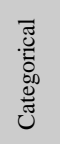 & 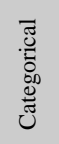 & 跣 & 节 & 这 & 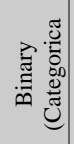 & 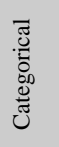 & $\begin{array}{l}\frac{U}{\overline{\underline{V}}} \\
\overline{\bar{z}}\end{array}$ & 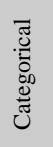 & $\begin{array}{l}\frac{\mathscr{E}}{\overline{\underline{n}}} \\
\frac{1}{\bar{z}}\end{array}$ & 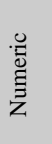 & 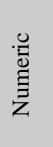 & 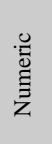 & 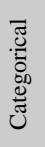 & 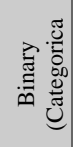 \\
\hline
\end{tabular}

The remainder of the paper is organized as follows: Section (2) focuses on the definition and features of data mining. Section (3) reviews of the neural network. Section (4) and section (5) will present definitions of naïve Bayes classifier and Logistic regression analysis. The technique C5.0 will be discussed in section (6). The discussion of data and interpretation of it will be in section (7). Section (8) demonstrates the experimental results to show the effectiveness of each model, then the conclusion will present in (9).

\section{DATA MINING OVERVIEW}

The definition of Data Mining or Knowledge Discovery in Databases is the action that extracts some new important information contained in large databases. The target of data mining is to find unexpected characteristics, hidden features or other unclear relationships in the data based on techniques' combination. Today, many applications in a wide and various ranges of business founded and worked in this regulation [7].

In 1996, U. Fayyad, G. Shapiro defined the general knowledge discovery process as an interactive and iterative process involving more or less the following steps: understanding the application field, data selecting, preprocessing and cleaning data, integration of data, data reduction and transformation, selecting algorithms of data mining, interpretation and description of the results and using the discovered knowledge [2]. In fact, the data mining can be classified into two categories descriptive and predictive.

Actually, in the recent years, data mining is occupying great position of attention area in the society of business or banking because its elasticity in working with a large amount of data, and turning such data into clear information and knowledge $[16,26]$. Most of the people may be confused in understanding between the terms "knowledge discovery" and "data mining" in different areas. Knowledge discovery in databases is the process of identifying valid, novel, probably useful, and finally understandable patterns/models with data. On the other hand, data mining is a step in the knowledge discovery process consisting of particular data mining algorithms that under some acceptable computational efficiency limitations, finds patterns or models in data [21].

\section{MULTILAYER PERCEPTION NEURAL NETWORK}

Multilayer perception neural network (MLPNN) with backpropagation is the most popular artificial neural network architecture $[17,26]$. The MLPNN is known to be a powerful function approximation for prediction and classification problems.

Historically, this direction field started when neurophysiologist Warren McCulloch and mathematician Walter Pitts introduced a paper on how neurons might work in 1943. They found a model for simple neural network using electrical circuits [13]. They named this model 'threshold logic'. The model opened the door on the way for research into neural network to divide into two distinct approaches. One approach concentrated on biological processes in the brain, and the other focused on the application of neural networks to artificial intelligence $[3,24]$.

The most interesting in the field was renewed in 1982. John Hopfield introduced an approach to construct more useful machines using bidirectional lines. In 1986, with multiple layered neural networks appeared three independent groups of researchers, one of which included David Rumelhart, presented similar ideas, which are now, called back propagation networks because it distributes pattern recognition errors throughout the network. Hybrid networks used just two layers; these back-propagation networks use many. Neural networks are applied to data mining in Craven and Sahvlik (1997) [13, 28].

Figure 1 shows that the MLPNN structure is organized into layers of neuron's input, output and hidden layers. There is at least one hidden layer, where the actual computations of the network are processed. Each neuron in the hidden layer sums its input attributes $x_{i}$ after multiplying them by the strengths of the respective connection weights $w_{\mathrm{ij}}$ and computes its output $y_{j}$ using activation function (AF) of this sum. AF may range from a simple threshold function, or a sigmoid, hyperbolic tangent, or radial basis function [3].

$y_{i}=f\left(\sum w_{i j} x_{i}\right)$

Back-propagation (BP) is a common training technique for MLPNN. The available data set is normally divided into training and test subsets. BP works by presenting each input sample to the network where the output is computed by 
performing weighted sums and transfer functions. The sum of squared differences between the desired and asset value of the output neuron's $E$ is defined as:

$$
E=\frac{1}{2} \sum_{j}\left(y_{d j}-y_{j}\right)^{2}
$$

Where $y_{d j}$ is the desired value of an output neuron $j$, and $y_{j}$ is the output of that neuron.

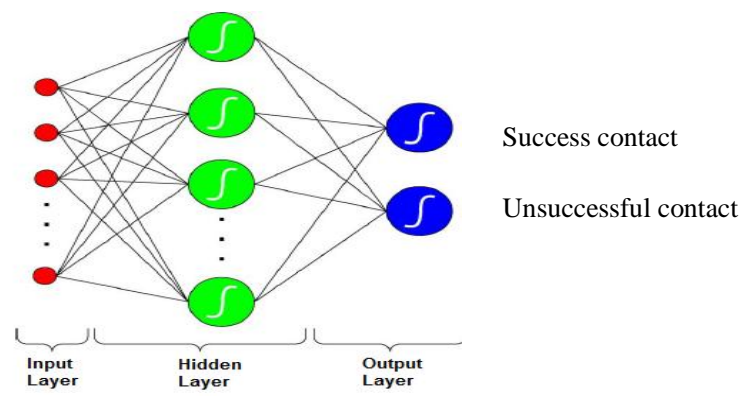

Fig 1. The structure of multilayer perceptron neural network.

Weights $w_{i j}$ in Equation (1), are adjusted to finding the minimum error $E$ of Equation (2) as fast, quickly as possible. $\mathrm{BP}$ applies a weight correction to reduce the difference between the network outputs and the desired ones; i.e., the neural network can learn, and can thus reduce the future errors. The performance of MLPNN depends on network parameters, the network weights and the type of transfer functions used [25].

When using MLPNN, three important issues need to be addressed; the selection of data samples for network training, the selection of an appropriate and efficient training algorithm and determination of network size. New algorithms for data portioning and effective training with faster convergence properties and fewer computational requirements are being developed [26]. However, the third issue is a more difficult problem to solve. It is necessary to find a network structure small enough to meet certain performance specifications. Pruning methods for improving the input-side redundant connections were also developed that resulted in smaller networks without degrading or compromising their performance [17].

Finally, MLPNN has many advantages, such as the good learning ability, less memory demand, suitable generalization, fast real-time operating, simple and convenient to utilize, suited to analyze complex patterns, and so on. Therefore, it has become a research hotspot in the past few years. On the other hand, there are some disadvantages like: the neural network requires high-quality data; variables must be carefully selected a priori, the risk of over-fitting, and requires a definition of architecture [13].

\section{NAÏVE BAYES CLASSIFIER}

Naïve Bayes (TAN) is one of the most effective and efficient classification algorithms. It is a one special case of a Bayesian network. The structure and parameters of the unconstrained Bayesian network would appear to be a logical means of improvement. However, (TAN) was found by Friedman (1997) as an easily outperforms such an unconstrained Bayesian network classifier on a huge sample of benchmark data sets. Bayesian classifiers are helpful in predicting the probability that a sample belongs to a particular class or grouping. This technique is useful for large databases because it is highly accurate and quickly in classification and fast to train with simple models and intuitive. It requires a small amount of training data to estimate the parameters (means and variances of the variables) necessary for classification, handles real and discrete data, also it can handle streaming data well. In another way, some often apparent disadvantages of Bayesian analysis are really not problems in practice. Any ambiguities in a prior choosing are generally not dangerous, since the various possible convenient priors usually do not disagree strongly within the regions of interest. Bayesian analysis is not limited to what is traditionally considered statistical data, but can be applied to any space of models [12].

A learner in classification learning problems, attempts to construct a classifier from a given set of training instances with class labels. Suppose that $n$ attributes are named as: $\left(A_{l}\right.$, $\left.A_{2}, \ldots, A_{n}\right)$. The instances are represented by a vector $\left(a_{1}, a_{2}, \ldots\right.$ , $a_{n}$ ), where $a i$ is the value of $A i$. Let $Y$ represents the class variable or the target attribute and $y$ represent the value of $Y$. A naïve Bayes classifier in this paper's data set of independent attributes is defined as:

$P\left(a_{i} \mid y\right)=\prod_{i=1}^{n} P\left(a_{i} \mid y\right)$

The structure of naïve Bayes on the database applied in this paper will be shown graphically in Figure 2. In this figure, the class node is the parent for each attribute node, but not exist any parent from attribute nodes. Naïve Bayes is easy to construct because the values of $P\left(a_{i} \mid y\right)$ can be easily estimated from training instances [8]

In addition, to improve the conditional independence assumption there exists one way, it is to enlarge the structure of naïve Bayes to represent explicitly attribute dependencies by adding arcs between attributes.

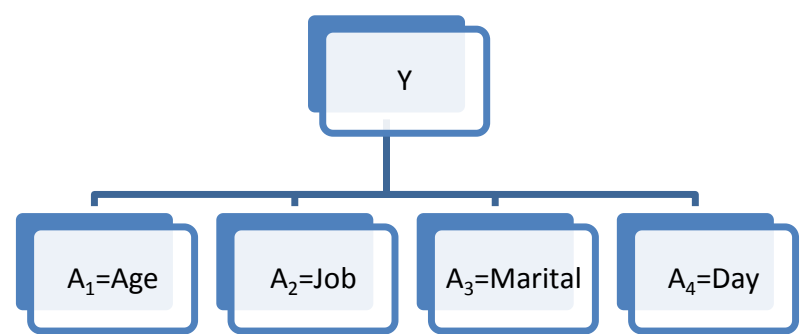

Fig 2. An example of naïve Bayes

Tree augmented naïve Bayes (TAN) is an extended tree such that a class node directly walks to all attribute nodes, also an attribute node can have only one parent from another attribute node. However, in TAN there is no limitation on the links between attribute nodes (except that they do not form any directed cycle) $[5,8]$. Figure 3 shows an example of TAN from the direct bank marketing database.

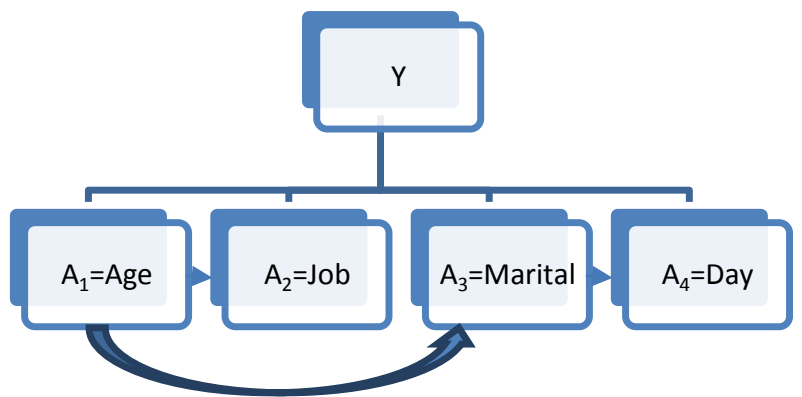

Fig 3. An example of TAN 


\section{LOGISTIC REGRESSION ANALYSIS}

The logistic regression (LR) model is very suitable for addressing issues of many kinds of data sets; it is provided sufficiently several and well-distributed samples. In addition, it is well suited for describing and testing hypotheses about relationships between a categorical outcome variable and one or more categories or continuous predictor attributes.

Furthermore, LR uses maximum probability estimation rather than the least squares estimation used in traditional multiple regression. Starting values of the predicted parameters are used and the probability that the sample came from a population with those parameters is computed. The values of the estimated parameters are adjusted iteratively until the greatest probability value of them is obtained. That is, maximum probability approaches try to find estimates of parameters that make the data observed "most likely" [4].

However, LR is an approach to learning functions of the form: $F: A \rightarrow Y$, or $P(Y \mid A)$ in the case where $\mathrm{Y}$ is discrete-valued as a target, and $A=\left(A_{l}, A_{2}, \ldots, A_{n}\right)$ is any attribute containing discrete, flag or continuous independent attributes. In the case of the bank, direct marketing data set $\mathrm{Y}$ is a flag attribute (yes or no) then the option of forward binomial procedure in the partitioned data is selected.

The logistic formulas are stated in terms of the probability that $Y=1$ (or yes), which is referred to as $P$. The probability that $\mathrm{Y}$ is 0 (or no) is $1-P[4,6]$.

$\ln \left(\frac{P}{1-P}\right)=w_{\circ}+w_{1} A$

Where $w_{\circ}+w_{1} A$ is the familiar equation for the regression line. Consequentially, LR assumes a parametric form the distribution $P(Y \mid A)$, after that directly estimates its parameters from the training data. The parametric model is [14]:

$P(Y=y e s \mid A)=\frac{\exp \left(w_{0}+\sum_{i=1}^{n} w_{i} A_{i}\right)}{1+\exp \left(w_{0}+\sum_{i=1}^{n} w_{i} A_{i}\right)}$

and

$P(Y=n o \mid A)=\frac{1}{1+\exp \left(w_{0}+\sum_{i=1}^{n} w_{i} A_{i}\right)}$

Where, $w_{0}$ is the constant of the equation and, $w_{i}$ is the coefficient of the predictor variables. The equation (4), which is known as a Logits (log odds) are the coefficients (the slope values) of the regression equation. The slope can be interpreted as the change in the average value of $\mathrm{Y}$, from one unit of change in $A$ [14].

Several advantages of LR such that: it is more robust because the independent variables don't have to be ordinarily distributed, or have equal variance in each group, it does not assume a linear relationship between the input attributes and dependent attribute, i.e., no linearity also it may handle nonlinear effects. Again, explicit interaction and power terms can be added, no homogeneity of variance, and the errors are not normally distributed. Additionally, there is no homogeneity of variance supposition normally distributed error terms are not assumed i.e., no normality; it does not require that the independents be an interval, and not require that the independents be unbounded.

Unfortunately, the advantages of logistic regression come at a cost because it requires much more data to achieve stable, meaningful results. With traditional regression, typically 20 data points per predictor are considered the lower bound. For logistic regression, at least 50 data points per predictor are necessary to achieve stable results.

\section{DECISION TREE MODEL TECHNIQUE}

Data mining techniques include many that should be in the circle of interest for financial people dealing with huge and complicated data sets. One of the most popular of the data mining techniques, decision trees, originated in the statistics' discipline $[9,15]$.

Decision tree algorithm partitions the data samples into two or more subsets so that the samples within each subset are more homogeneous than in the previous subset. This is a recursive process; the resulting two subsets (in binary decision tree) are then split again, and the process repeats until the homogeneity criterion is reached or until some other stopping, criterion is satisfied $[1,2]$.

As the name implies, this model recursively separates data samples into branches to construct a tree structure for improving the prediction accuracy. Each tree node is either a leaf node or decision node. All decision nodes have to split, testing the values of some functions of data attributes. Each branch of the decision node corresponds to a different outcome of the test as in Figure 4.

Historically, the book by Bremen et al. (1993) provided an introduction to decision trees that is still considered the standard resource on the topic. Two reasons for the popularity of decision tree techniques are the procedures are relatively straightforward to understand and explain, and the procedures address a number of data complexities, such as nonlinearly and interactions, that commonly occur in real data $[5,16]$.

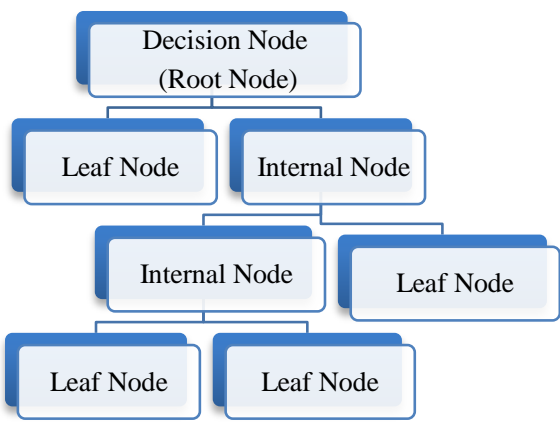

Fig 4. Illustrated example of a binary decision tree

The famous typical in decision trees is C5.0, which is a recently invented modeling algorithm, and it is an improved version of C4.5 and ID3 algorithms. C5.0 is a commercial product designed by Rule Quest Research Ltd Pty to analyze huge data sets and is implemented in SPSS Clementine workbench data mining software [2, 5, 21].

The tree of C5.0 uses common splitting algorithms includes entropy based on information gain. The model works by splitting the sample based on the attribute that provides the maximum information gain. Each sub sample defined by the first split is then split again, usually based on a different attribute, and the process repeats until the subsamples cannot be split any further. Finally, the low-level splits are reexamined, and those that do not contribute significantly to the value of the model are removed or pruned.

C5.0 model is quite robust in the presence of problems such as missing data and large numbers of input fields. It usually does not require long training times to estimate. In addition, C5.0 models tend to be easier to understand than some other model types, since the rules derived from the model have a very straightforward interpretation. Furthermore, C5.0 offers the 
powerful boosting method to increase accuracy of classification. C5.0 uses entropy as a measure of purity, which is based on an information gain [16].

The entropy is a commonly used measure in information gain and defined as that characterizes of the (im) purity of an arbitrary collection of data. If $Y$ containing only flag classes (yes and no) of some target concept, the entropy of set $Y$ relative to this simple, binary classification is defined as:

$\operatorname{entropy}(Y)=\left(\sum_{i=1}^{n}-p_{i} \log _{2} p_{i}\right)$

Where $p_{i=1}$ is the proportion of yes classes in $m y$ and $p_{i=2}$ is the proportion of no classes in $Y$, where $n$ has only two options in this database of the bank direct marketing used in this paper. In addition, the entropy is 1 (at its maximum!) when the collection contains an equal number of yes and no classes. If the collection contains unequal numbers of yes and no, the entropy is in between 0 and 1 . Figure 5 shows the form of the entropy function relative to a binary classification, as $p_{\text {yes }}$ varies between 0 and 1 .

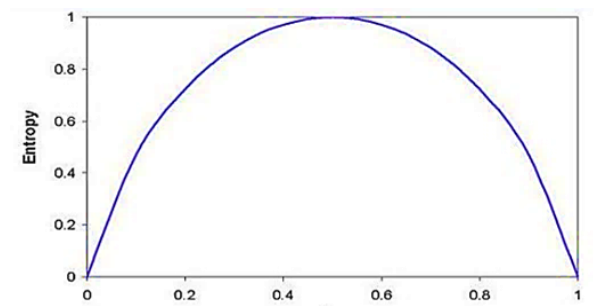

Fig 5: The entropy function relative to a binary classification, as the proportion of yes

If the entropy is a measure of the impurity in a collection of training classes, then the measure of the effectiveness of an attribute in classifying the training data is called information gain, which is simply the expected reduction in entropy caused by partitioning the classes according to this attribute. More precisely, the information gain, Gain $(Y, A)$ of an attribute $A$, relative to a collection of classes $Y$ is defined as:

$\operatorname{gain}(Y, A)=\operatorname{entropy}(Y)-\sum_{v \in \operatorname{values}(A)} \frac{\left|Y_{v}\right|}{|Y|} \operatorname{entropy}\left(Y_{v}\right)$

Where Values (A) are the set of all possible values for attribute $A$, and $Y_{v}$ is the subset of $Y$ for which attribute $A$ has value $v$ (i.e., $Y_{v}=\{\mathrm{y} \in Y \mid A(y)=v\}$ ) [15].

Furthermore, the first term in the equation for Gain is just the entropy of the original collection $Y$, and the second term is the predictable value of the entropy after $Y$ is partitioned using attribute $A$. The expected entropy described by this second term is simply the sum of the entropies of each subset $Y_{v}$, weighted by the fraction of examples $\left|Y_{v}\right| /|Y|$ that belongs to $Y_{v}$.

Gain $(Y, A)$ is therefore, the expected reduction in entropy caused by knowing the value of attribute $A$. Put another way, $\operatorname{Gain}(Y, A)$ is the information provided about the target attribute value, given the value of some other attribute $A$. The value of $\operatorname{Gain}(Y, A)$ is the number of bits saved when encoding the target value of an arbitrary member of $Y$, by knowing the value of attribute $A$ [15].

The process of selecting a new attribute and partitioning the training examples is repeated for each non-terminal descendant node, this time using only the training examples connected to that node. Attributes that have been included higher in the tree are excluded, so that any given attribute can appear at most once along any path through the tree. This process continues for each new leaf node until either of two conditions is met: every attribute has already been included along this path through the tree, or the training examples associated with this leaf node all have the same target value (i.e., their entropy is zero) [23].

Boosting, winnowing and pruning are three methods used in the C5.0 tree construction; they propose to build the tree with the right size [26]. They increase the generalization and reduce the over fitting of the decision tree model.

\section{DATA SET DISCRIPTION}

This paper employed the bank direct marketing data set from the University of California at Irvine (UCI) Machine Learning Repository have been used to evaluate the performances of the multilayer perception neural network (MPLNN), Naïve Bayes (TAN), logistic regression (LR), and C5.0 decision tree classification model. The bank direct marketing data set used here was collected by S. Moro, R. Laureano and P. Cortez [22]. The data is related to direct marketing campaigns of a Portuguese banking institution. The marketing campaigns were based on phone calls. Often, more than one contact with the same client was required, in order to access if the product (bank term deposit) were (or not) subscribed. The bank direct marketing data set contains (45211) number of samples with (17) attributes without missing values [10, 22].

The characteristics of data set composed of two kinds: nominal and numeral attributes, as shown in Table 2. This table shows that three kinds of attributes; Numerical, which are in range type for all of them like (Age, Balance, Day, Duration, campaign, Pdays, and Previous), Categorical are in set type as the attributes (Job, Marital, Education, Contact, Month, Poutcome), and Binary categories are all the attributes that represented as yes or no in their classes; for example, the attributes (Default, Housing, Loan, Output).

The column headed Attributes illustration is presenting the number classes for each attribute and the relation with its name. In the second attribute named Job, there exist many kinds of jobs belonging to this attribute as (admin, unknown, unemployed, management, housemaid, entrepreneur, student, blue-collar, self-employed, retired, technician, and services). The attribute Marital can be illustrated in classes as (married, divorced, and single) where the class divorce means divorced or widowed. The Education classes are divided into unknown, secondary, primary, and tertiary; however, in attributes Default, Housing, Loan, and the output attribute has only two classes (yes, and no). The contact communication classes in the Contact attribute are: unknown, telephone, and cellular. Clearly, in the attribute Month the classes are month's names Jan, Feb, etc. The attribute Poutcome presents the outcome of the previous marketing campaign like: unknown, other, failure, and success. The last column in table 2 introduces the duration for each range in the numerical kind of attributes; for example, Age attribute has (18:95) in duration; that means all ages for customers or samples range between 18 and 95 years, also; the average yearly balance is in between -8019 and 102127. 
Table 2. ATTRIBUTES DESCRIPTION

\begin{tabular}{|c|c|c|c|c|c|}
\hline \# & Attributes & Kind & Type & Attributes illustration & Domain \\
\hline 1 & Age & Numeric & Range & $\mathrm{NaN}$ & $18: 95$ \\
\hline 2 & Job & Categorical & Set & $\begin{array}{l}\text { ('admin.','unknown','unemployed','management','housemaid','entrepreneur','student','blue- } \\
\text { collar','self-employed','retired','technician','services') }\end{array}$ & $\mathrm{NaN}$ \\
\hline 3 & Marital & Categorical & Set & marital status ('married','divorced','single'; note: 'divorced' means divorced or widowed) & $\mathrm{NaN}$ \\
\hline 4 & Education & Categorical & Set & ('unknown','secondary','primary','tertiary') & $\mathrm{NaN}$ \\
\hline 5 & Default & Binary (Categorical) & Flag & has credit in default? (binary: 'yes','no') & $\mathrm{NaN}$ \\
\hline 6 & Balance & Numeric & Range & average yearly balance, in euros & -8019: 102127 \\
\hline 7 & Housing & Binary (Categorical) & Flag & has housing loan? (binary: 'yes','no') & $\mathrm{NaN}$ \\
\hline 8 & Loan & Binary (Categorical) & Flag & has personal loan? (binary: 'yes','no') \# related with the last contact of the current campaign & $\mathrm{NaN}$ \\
\hline 9 & Contact & Categorical & Set & contact communication type (categorical: 'unknown','telephone','cellular') & $\mathrm{NaN}$ \\
\hline 10 & Day & Numeric & Range & last contact day of the month & $1: 31$ \\
\hline 11 & Month & Categorical & Set & last contact month of year (categorical: 'jan', 'feb', 'mar', ..., 'nov', 'dec') & $\mathrm{NaN}$ \\
\hline 12 & Duration & Numeric & Range & last contact duration, in seconds & $0: 4918$ \\
\hline 13 & Campaign & Numeric & Range & number of contacts performed during this campaign and for this client (includes last contact) & $1: 63$ \\
\hline 14 & Pdays & Numeric & Range & $\begin{array}{l}\text { number of days that passed by after the client was last contacted from a previous campaign (- } \\
1 \text { means client was not previously contacted) }\end{array}$ & $-1: 871$ \\
\hline 15 & Previous & Numeric & Range & number of contacts performed before this campaign and for this client & $0: 275$ \\
\hline 16 & Poutcome & Categoricasl & Set & outcome of the previous marketing campaign (categorical:'unknown','other','failure','success') & $\mathrm{NaN}$ \\
\hline 17 & Output & Binary (Categorical) & Flag & Output variable (desired target):y-has the client subscribed a term deposit? (binary: 'yes','no') & $\mathrm{NaN}$ \\
\hline
\end{tabular}

In the same context, the month's days of course have ranged from 1 to 31, and the last contact duration in seconds in the attribute of Duration is in between 0 to 4918 seconds. The attribute Campaign shows in its domain the number of contacts performed during this campaign, and for this, client (includes last contact) is in the interval from 1 to 63; however, the domain ranged between -1 to 871 is representing the number of days that passed by after the client was last contacted from a previous campaign ( -1 means client was not previously contacted) in the attribute Pdays. Last but not least, the attribute Previous presents the number of contacts performed before this campaign and for this client, its domain from 0 to 275 .

In fact, methods for analyzing and modeling data can be split into two groups: supervised learning and unsupervised learning. The supervised learning requires input data that has both predictor (independent) attributes and a target (dependent) attribute whose value is to be estimated. In addition, the process learns how to model (predict) the value of the target attribute based on predictor attributes. The famous examples of supervised learning are decision trees, and neural networks. Actually, the supervised learning is suitable for analysis dealing with the prediction of some attribute [24].

On the other hand, unsupervised learning instead of identifying a target (dependent) attribute treats all the attributes equally. In this kind of methods, the goal is searching about patterns, groupings or other ways to distinguish the data, which may lead to the understanding of data relations; not to predict the value of an attribute like the previous kind of analyzing method. The examples of unsupervised learning are: correlation, statistical measures, and cluster analysis [21]. This paper is used supervised learning of data analysis to reach to the best prediction for the attribute $Y$ (Output), which is the target. The objective is to examine the performance of MLPNN, TAN, LR and C5.0 models on a real-world data of bank deposit subscription and increasing the campaign effectiveness by identifying the main characteristics that affect the success (the deposit subscribed by the client).

Table 3 shows that a classification for all attributes, they are divided into two parts; each one has some of the attributes with ranges (for numerical attributes) and classes (for category and binary categorical attributes), and also the percentages for every class or interval in the range of attributes are calculated. By these percentages, the most common age category for the customers in this data set of the bank is inside the interval from 30 to 40 years in the attribute of Age by $40 \%$, as well; the public job in these samples is Blue-collar in the attribute of Job by $22.47 \%$. In the same context, the highest percentage is $60 \%$ for married customers in the attribute of Marital, and most of them learnt to the secondary class by ratio $51 \%$ in the Education attribute.

\section{TABLE 3 PART (1) BANK DIRECT MARKETING ATTRIBUTES' VALUES PERCENTAGES}

\begin{tabular}{|c|c|c|c|c|c|c|c|c|c|c|c|c|c|c|c|}
\hline \multicolumn{2}{|l|}{ Age } & \multicolumn{2}{|l|}{ Job } & \multicolumn{2}{|l|}{ Marital } & \multicolumn{2}{|l|}{ Education } & \multicolumn{2}{|c|}{ Default } & \multicolumn{2}{|c|}{ Balance } & \multicolumn{2}{|c|}{ Housing } & \multicolumn{2}{|l|}{ Loan } \\
\hline Range & $\%$ & Class & $\%$ & Class & $\%$ & Class & $\%$ & Class & $\%$ & Range & $\%$ & Class & $\%$ & Class & $\%$ \\
\hline$[18,30)$ & $12 \%$ & Admin & $11 \%$ & Married & $60 \%$ & Primary & $16 \%$ & Yes & $2 \%$ & {$[-8019,10,000)$} & $98.17 \%$ & Yes & $56 \%$ & Yes & $16 \%$ \\
\hline$[30,40)$ & $40 \%$ & Entrepreneur & $3 \%$ & Single & $28 \%$ & Secondary & $51 \%$ & No & $98 \%$ & {$[10,000,20,000)$} & $1.4 \%$ & No & $44 \%$ & No & $84 \%$ \\
\hline$[40,50)$ & $25.47 \%$ & Blue-collar & $22 \%$ & Divorced & $12 \%$ & Tertiary & $29 \%$ & & & {$[20,000,30,000)$} & $.312 \%$ & & & & \\
\hline$[50,60)$ & $18.6 \%$ & Retired & $5 \%$ & & & Unknown & $4 \%$ & & & {$[30,000,40,000)$} & $0.051 \%$ & & & & \\
\hline$[60,70)$ & $2.7 \%$ & Technician & $17 \%$ & & & & & & & {$[40,000,50,000)$} & $.022 \%$ & & & & \\
\hline$[70,80)$ & $0.94 \%$ & Student & $2 \%$ & & & & & & & {$[50,000,60,000)$} & $.022 \%$ & & & & \\
\hline$[80,90)$ & $0.27 \%$ & Management & $21 \%$ & & & & & & & {$[60,000,70,000)$} & $.008 \%$ & & & & \\
\hline$[90,100)$ & $0.02 \%$ & Self- & $3.74 \%$ & & & & & & & {$[70,000,80,000)$} & $.0022 \%$ & & & & \\
\hline
\end{tabular}


Specificity $=\frac{T N}{T N+F P}$

Figure 6 shows the component nodes of the proposed stream. The stream is implemented in SPSS Clementine data mining workbench using Intel ${ }^{\circledR}$ core ${ }^{\text {TM }} 2$ Duos, CPU with 1.83
GHz. Clementine uses client/server architecture to distribute requests for resource-intensive operations with powerful server software, resulting in faster performance on larger data sets [10]. The software offers many modeling techniques, such as prediction, classification, segmentation, and association detection algorithms.

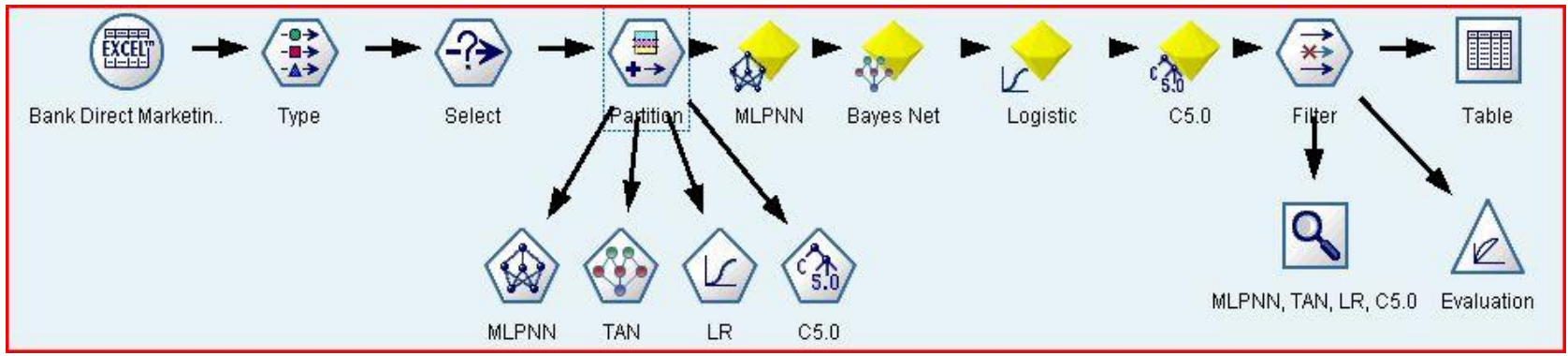

Fig 6. A data mining stream for the prediction of the bank deposit subscription using MLPNN, TAN, LR, and C5.0 respectively.

Bank direct marketing data set node is connected directly to an EXCEL sheet file that contains the source data. The data set was explored as ordinal data types.

The type node specifies the field metadata and properties that are important for modeling and other work in Clementine. These properties include specifying a usage type, setting options for handling missing values (the used data set in this paper has not been missing values to handle), as well as setting the role of an attribute for modeling purposes; input or output. As previously stated, the first 16 attributes in table 2 are defined as input attributes and the output attribute (y) is defined as a target.

The MLPNN classifier node is trained using the pruning method. It begins with a large network and removes the weakest neurons in the hidden and input layers as training proceeds. To prevent over training, the portion of the training subset has been used as a validation subset. The network is trained on the rest of the training subset, and accuracy is estimated based on the validation subset. The stopping criterion is set based on time; the network is trained for one minute.

However, the training process may be interrupted at any point to save the network model with the best accuracy achieved so far. By using the direct-marketing campaigns of a Portuguese banking institution data set, the resulting structure consists of four layers; one input with 48 neurons, two hidden layers: first hidden layer has 20 neurons and the second hidden layer has 15 neurons, and the output layer with one neuron. The prediction accuracies of training and test samples are $90.79 \%$ and $90.02 \%$ respectively.

Bayesian Network (TAN) node is a graphical model that displays attributes (often referred to as nodes) in a data set and the probabilistic, or conditional, independence between them. Fundamental relationships between nodes may be represented by a Bayesian network; however, the links in the network (also known as arcs) do not necessarily represent direct cause and effect. It is building a probability model by combining observed and recorded evidence with "common sense" realworld knowledge to establish the likelihood of occurrences by using seemingly independent attributes. In the current Clementine 12.0 release, the node focuses on Tree Augmented Naïve Bayes (TAN), (in this paper), and Markov Blanket networks that are primarily used for classification.
The Tree Augmented Naïve Bayes model (TAN) creates a simple Bayesian network model that is an improvement over the standard Naïve Bayes model. This is because it allows each predictor to depend on another predictor in addition to the target variable, thereby increasing the classification accuracy. The prediction accuracies of training and test samples are $89.16 \%$ and $88.75 \%$ respectively. Figure 7 shows that the resulted Bayesian network (TAN).

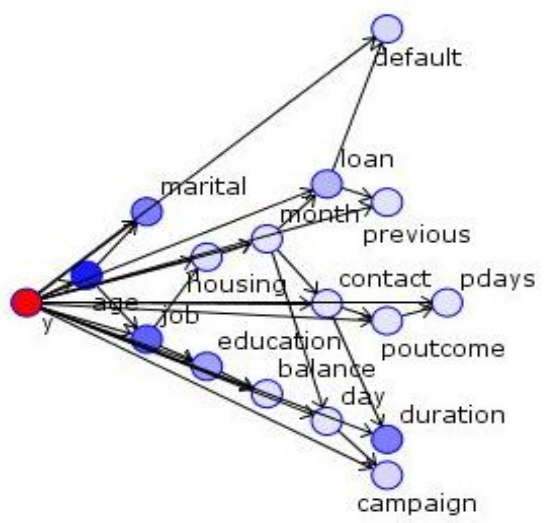

Type

Predictors

Targets

Importance

0.25

0.20

0.15

0.10

0.05

0.00

Fig 7. The Bayesian network TAN

Logistic regression (LR) node, also known as nominal regression, is a statistical technique for classifying records based on values of input attributes. It is analogous to linear regression but takes a categorical target field instead of a numeric one. LR contains two kinds for classification: binomial models (for targets with two discrete categories as in our data set) and multinomial models (for targets with more than two categories). It is working by building a set of equations that relate the input field values to the probabilities associated with each of the output field categories. Once the model is generated, it can be used to estimate probabilities for new data. For each record, a probability of membership is computed for each possible output category. The target category with the highest probability is assigned as the predicted output value of that record. A binomial model is used because the target has two distinct classes (yes and no). In the results of prediction, the prediction accuracies of 
training and test samples are $90.09 \%$ and $90.43 \%$ respectively.

C5.0 node is trained and tested using a simple model with the partitioned data. The minimum number of samples per node is set to be 2 and the decision tree with 12 in depth. It will examine the importance rate of the predictors before starting to build the model by winnow attribute's option. Predictors that are found to be irrelevant are then removed from the model-building process [2]. The prediction accuracies of training and test samples are $93.23 \%$ and $90.09 \%$ respectively.

Filter, Analysis and Evaluation nodes are used to select and rename the classifier outputs in order to compute the performance statistical measures and to graph the evaluation charts. Table 5 shows the numerical illustration of the importance of the attributes with respect to models MLPNN, TAN, LR and C5.0.

TABLE 5 THE IMPORTANCE OF ATTRIBUTES RELATED TO THE MLPNN AND C5.0

\begin{tabular}{|c|c|c|c|c|c|c|c|c|c|c|c|c|c|c|c|c|}
\hline Models & \multicolumn{16}{|c|}{ Importance } \\
\hline & $\underset{\pi}{\infty}$ & $\stackrel{0}{\circ}$ & 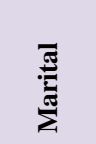 & : & 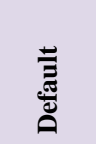 & 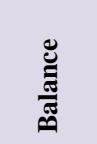 & $\begin{array}{l}.00 \\
0 \\
0 \\
0 \\
0 \\
0\end{array}$ & ‡్ & 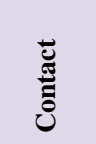 & $\vec{\Xi}$ & 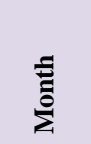 & ڤ્气 & 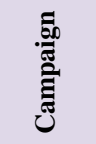 & $\sum_{0}^{\infty}$ & 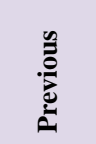 & 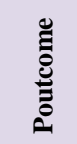 \\
\hline MLPNN & 0.0223 & 0.0504 & 0.021 & 0.0247 & 0.0062 & 0.0083 & 0.0226 & 0.0174 & 0.0555 & 0.0478 & 0.1762 & 0.326 & 0.0198 & 0.0376 & 0.0142 & 0.15 \\
\hline TAN & 0.2921 & 0.1902 & 0.1482 & 0.0955 & 0.018 & 0.0056 & 0.0107 & 0.0674 & 0 & 0 & 0 & 0.148 & 0.0217 & 0 & 0.0024 & 0 \\
\hline LR & ------ & 0.0344 & 0.0139 & 0.0136 & ----- & ------ & 0.0613 & 0.0172 & 0.0807 & 0 & 0.0531 & 0.499 & 0.0346 & ------ & 0.0131 & 0.179 \\
\hline C5.0 & 0 & 0.0186 & 0.0301 & 0 & ----- & 0.0237 & 0 & 0.0152 & 0 & 0 & & 0.722 & 0.0206 & 0.0095 & 0.0072 & 0.153 \\
\hline
\end{tabular}

The table illustrates that the attribute Duration is the most important for three examined models. In MLPNN, the ratio is $0.339,0.499$ for LR and in C5.0 is 0.722 that they are highest ratios among all the other attributes; however, the attribute Age is nominated by TAN with ratio 0.292 . The attribute Default is removed by LR and C5.0, also Age, Balance, Pdays attributes are removed by LR only because it is trivial or not has any degree of importance with respect these models. However, some attributes are not removed; nevertheless, they measured from zero because their importance is very low and rounded to zero, such as Contact, Day, Month in models TAN and C5.0, Pdays, Poutcome attributes only in TAN and Day in LR again rounded to zero. These all ratios are illustrated in Figure 8 .

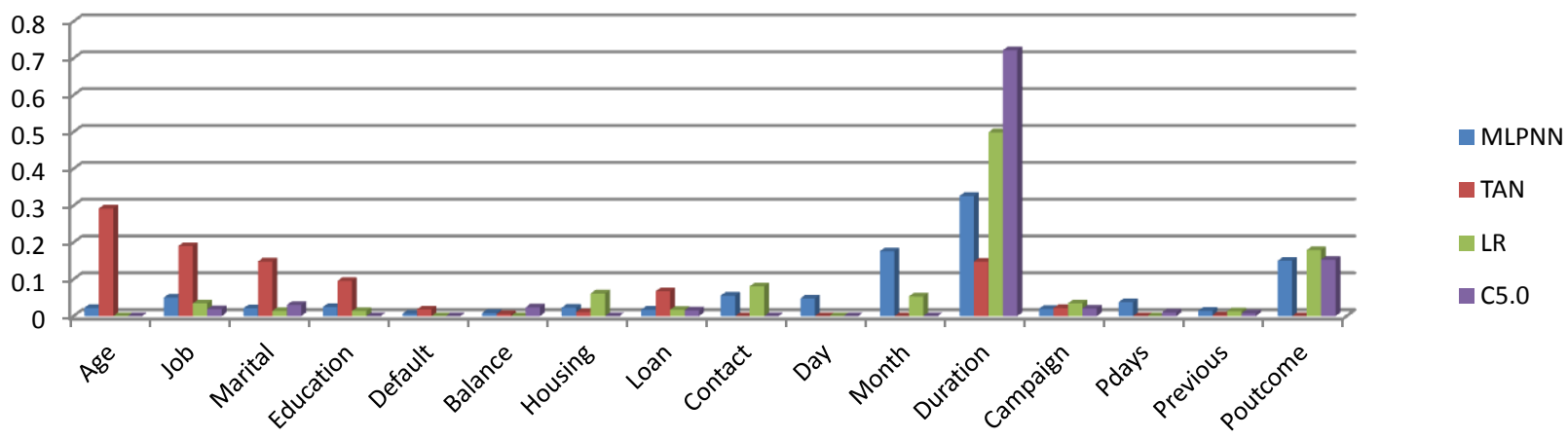

Fig 8. The most importance of attributes based on MLPNN, TAN, LR, and C5.0

Figure 9 shows the cumulative charts of the four models for training and test subsets. The higher lines indicate better models, especially on the left side of the chart. The four curves are same for the test subset and almost identical to the training one. This figure shows that MLPNN line crowed LR

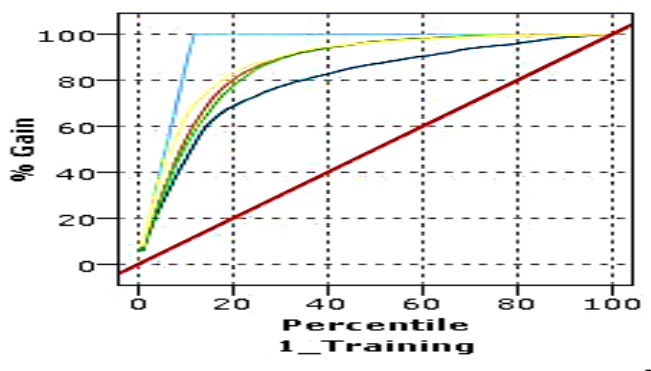

and C5.0 lines to reach the best line in the training subsets in some positions; even so, TAN leaves them and individually stayed alone. In the same contest, the success is observed for the alike three models MLPNN, LR, and C5.0 in the testing subset and TAN stayed far from them.

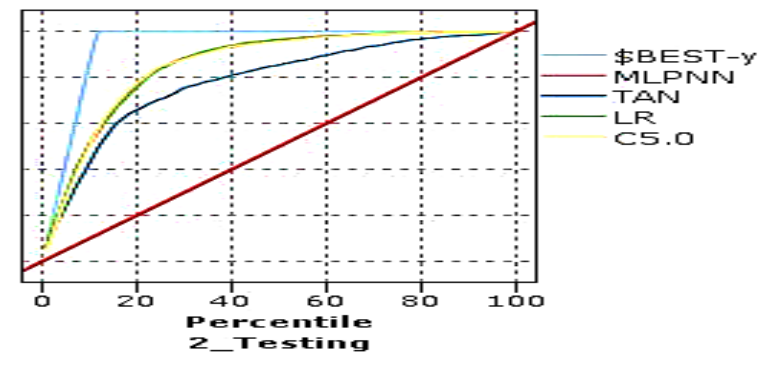

Fig 9. The cumulative gains charts of the four models for training and test subsets. 
The predictions of all models are compared to the original classes to identify the values of true positives, true negatives, false positives and false negative. These values have been computed to construct the confusion matrix as tabulated in
Table 6 where each cell contains the raw number of cases classified for the corresponding combination of desired and actual classifier outputs.

TABLE 6 The Confusion Matrices of MLPNN, TAN, LR and C5.0 Models for Training and Testing Subsets

\begin{tabular}{|c|c|c|c|c|c|c|}
\hline \multirow{2}{*}{ Model } & \multicolumn{3}{|c|}{ Training Data } & \multicolumn{3}{|c|}{ Testing Data } \\
\hline & Desired output & Yes & No & Desired output & Yes & No \\
\hline \multirow{2}{*}{ MLPNN } & Yes & $\mathrm{TP}=1,771$ & $\mathrm{FP}=1,941$ & Yes & $\mathrm{TP}=719$ & $\mathrm{FP}=858$ \\
\hline & No & $\mathrm{FN}=926$ & $\mathrm{TN}=26,951$ & No & $\mathrm{FN}=437$ & $\mathrm{TN}=11,608$ \\
\hline \multirow{2}{*}{ TAN } & Yes & 1,374 & 2,338 & Yes & 535 & 1,042 \\
\hline & No & 1,085 & 26,792 & No & 490 & 11,555 \\
\hline \multirow{2}{*}{ LR } & Yes & 1,270 & 2,442 & Yes & 578 & 999 \\
\hline & No & 689 & 27,188 & No & 304 & 11,741 \\
\hline \multirow{2}{*}{ C5.0 } & Yes & 2,258 & 1,454 & Yes & 740 & 837 \\
\hline & No & 684 & 27,193 & No & 513 & 11,532 \\
\hline
\end{tabular}

The values of the statistical parameters (sensitivity, specificity and total classification accuracy) of the four models were computed and presented in Table 7. Accuracy, Sensitivity and Specificity approximate the probability of the positive and negative labels being true. They assess the usefulness of the algorithm on a single model. By using the results shown in Table 7, it can be seen that the sensitivity, specificity and classification accuracy of all models has achieved 94.92\% success of training samples.

In this table, MLPNN model has accuracy $90.92 \%$ for training samples and $90.49 \%$ of tested samples, $89.16 \%$ and 88.75 for training and testing samples according to TAN. The classification accuracy of LR model is $90.09 \%$ and $90.43 \%$ for training and testing samples, also C5.0 model has $93.23 \%$ of training samples and $90.09 \%$ of testing samples, and so on.

The highest value in training samples is appeared for C5.0; nevertheless, the highest percentage of testing is in the cell of MLPNN for accurate classification. Simultaneously, the sensitivity analysis takes the highest percentage in C5.0 of the training samples $76.75 \%$; however, for testing samples the highest value is $65.53 \%$ for LR.

\section{TABLE 7 PERCENTAGES OF THE STATISTICAL MEASURES OF MLPNN, TAN, LR AND C5.0 FOR TRAINING AND TESTING SUBSETS}

\begin{tabular}{|l|l|l|l|l|}
\hline Model & Partition & Accuracy & Sensitivity & Specificity \\
\hline \multirow{2}{*}{ MLPNN } & Training & $90.92 \%$ & $65.66 \%$ & $93.28 \%$ \\
\cline { 2 - 5 } & Testing & $90.49 \%$ & $62.20 \%$ & $93.12 \%$ \\
\hline \multirow{2}{*}{ TAN } & Training & $89.16 \%$ & $55.87 \%$ & $91.97 \%$ \\
\cline { 2 - 5 } & Testing & $88.75 \%$ & $52.19 \%$ & $91.73 \%$ \\
\hline \multirow{2}{*}{ LR } & Training & $90.09 \%$ & $64.83 \%$ & $91.76 \%$ \\
\cline { 2 - 5 } & Testing & $90.43 \%$ & $65.53 \%$ & $92.16 \%$ \\
\hline \multirow{2}{*}{ C5.0 } & Training & $93.23 \%$ & $76.75 \%$ & $94.92 \%$ \\
\cline { 2 - 5 } & Testing & $90.09 \%$ & $59.06 \%$ & $93.23 \%$ \\
\hline
\end{tabular}

Last but not least, the specificity measure has C5.0 with the highest values in training samples $94.92 \%$ and $93.23 \%$ for testing samples. From the previews, C5.0 is the best in accuracy, sensitivity, and specificity analysis of training samples; however, the MLPNN is the best for accuracy; LR takes the best percentage for sensitivity, and C5.0 return to be the best in specificity analysis of testing samples.

\section{CONCLUSION}

Bank direct marketing and business decisions are more important than ever for preserving the relationship with the best customer. To success and survival, the business there is a need for customer care and marketing strategies. Data mining and predictive analytics can provide help in such marketing strategies. Its applications are influential in almost every field containing complex data and large procedures. It has proven the ability to reduce the number of false positives and falsenegative decisions. This paper has been evaluating and comparing the classification performance of four different data mining techniques' models MPLNN, TAN, LR and C5.0 on the bank direct marketing data set to classify for bank deposit subscription. The purpose is increasing the campaign effectiveness by identifying the main characteristics that affect the success (the deposit subscribed by the client). The classification performances of the four models have been using three statistical measures; Classification accuracy, sensitivity and specificity. This data set has partitioned into training and test by the ratio $70 \%$ and $30 \%$, respectively. Experimental results have shown the effectiveness of models. C5.0 has achieved slightly better performance than MLPNN, LR and TAN. Importance analysis has shown that attribute "Duration" in C5.0, LR, and MLPNN models have achieved the most important attribute; however, the attribute Age is the only assessed as more important than the other attributes by TAN.

\section{REFERENCES}

[1] A. Floares., A. Birlutiu. "Decision Tree Models for Developing Molecular Classifiers for Cancer Diagnosis". WCCI 2012 IEEE World Congress on Computational Intelligence June, 10-15, 2012 - Brisbane, Australia.

[2] Adem Karahoca, Dilek Karahoca and Mert Şanver, "Data Mining Applications in Engineering and Medicine", ISBN 978-953-51-0720-0, In Tech, August 8, 2012.

[3] B. Chaudhuri and U. Bhattacharya." Efficient training and improved performance of multilayer perceptron in 
pattern classification". Neuro computing, 34, pp11-27, September 2000

[4] D.G. Kleinbaum and M. Klein, Logistic Regression, Statistics for Biology and Health, DOI 10.1007/978-14419-1742-31, Springer Science Business Media, LLC 2010 .

[5] Derrig, Richard A., and Louise A. Francis, "Distinguishing the Forest from the TREES: A Comparison of Tree-Based Data Mining Methods," Variance 2:2, 2008, pp. 184-208.

[6] Domínguez-Almendros S. "LOGISTIC REGRESSION MODELS". Allergol Immunopathol (Madr). 2011. doi:10.1016/j.aller.2011.05.002.

[7] Eniafe Festus Ayetiran, "A Data Mining-Based Response Model for Target Selection in Direct Marketing", I.J.Information Technology and Computer Science, 2012, $1,9-18$.

[8] Harry Zhang., Liangxiao Jiang., Jiang Su. "Augmenting Naïve Bayes for Ranking". Published in: Proceeding of ICML '05 Proceedings of the 22nd international conference on Machine learning Pages 1020 - 1027, 2005 .

[9] Ho, T.B. (nd). "Knowledge Discovery and Data Mining Techniques and Practice". 2006, Available on: www.netnam.vn/unescocourse/knowlegde/knowfrm.htm

[10] http://archive.ics.uci.edu/ml (The UCI Machine Learning Repository is a collection of databases).

[11] http://en.wikipedia.org/wiki/Direct_marketing. Wikipedia has a tool to generate citations for particular articles related to direct marketing.

[12] http://en.wikipedia.org/wiki/Naïve_Bayes_classifier Wikipedia has a tool to generate citations for particular articles related to Naïve Bayes classifier.

[13] http://en.wikipedia.org/wiki/Neural_network\#History of the neural network analogy Wikipedia has a tool to generate citations for particular articles related to Neural Network.

[14] http://rimarcik.com/en/navigator/nlog.html Personale web site introducing many statistics tools illustrated by Marian Rimarcik.

[15] http://www2.cs.uregina.ca/ dbd/cs831/index.html Knowledge Discovery in Databases 2012.

[16] J. W. Han and M. Kamber. Data mining concepts and techniques, The $2^{\text {nd }}$ edition, Morgan Kaufmann Publishers, San Francisco, CA, 2006.
[17] L. Ma and K. Khorasani. "New training strategy for constructive neural networks with application to regression problems". Neural Networks, 17,589-609, 2004.

[18] O'guinn, Thomas." Advertising and Integrated Brand Promotion". Oxford Oxfordshire: Oxford University Press. p. 625. ISBN 978-0-324-56862-2. , 2008.

[19] Ou, C., Liu, C., Huang, J. and Zhong, N. 'One Data mining for direct marketing', Springer-Verlag Berlin Heidelberg, pp. 491-498., 2003.

[20] Petrison, L. A., Blattberg, R. C. and Wang, P. 'Database marketing: Past present, and future', Journal of Direct Marketing, 11, 4, 109-125, 1997.

[21] R. Nisbet, J. Elder and G. Miner. Handbook of statistical analysis and data mining applications. Academic Press, Burlington, MA, 2009.

[22] S. Moro, R. Laureano and P. Cortez. Using Data Mining for Bank Direct Marketing: An Application of the CRISP-DM Methodology. In P. Novais et al. (Eds.), Proceedings of the European Simulation and Modelling Conference - ESM'2011, pp. 117-121, Guimarães, Portugal, October, 2011

[23] Su-lin PANG, Ji-zhang GONG, C5.0 Classification Algorithm and Application on Individual Credit Evaluation of Banks, Systems Engineering - Theory \& Practice, Volume 29, Issue 12, Pages 94-104, December 2009.

[24] T. Munkata, "Fundamentals of new artificial intelligence," $2^{\text {nd }}$ edition, London, Springer-Verlag, 2008.

[25] TIAN YuBo, ZHANG XiaoQiu, and ZHU RenJie. "Design of Waveguide Matched Load Based on Multilayer Perceptron Neural Network". Proceedings of ISAP, Niigata, Japan 2007.

[26] Tom M. Mitchell. "Machine Learning”. Copyrightc 2005-2010. Second edition of the textbook. Chapter 1, all rights reserved. January 19, 2010 McGraw Hill. 119 ACM 2005, ISBN 1-59593-180-5.

[27] Freeman WJ. "The physiology of perception". University of California, Berkeley. Sci Am. 1991 Feb;264(2):78-85.

[28] Craven MW, Shavlik JW. "Understanding time series networks: a case study in rule extraction". Int J Neural Syst. 1997 Aug;8(4):373-84. 